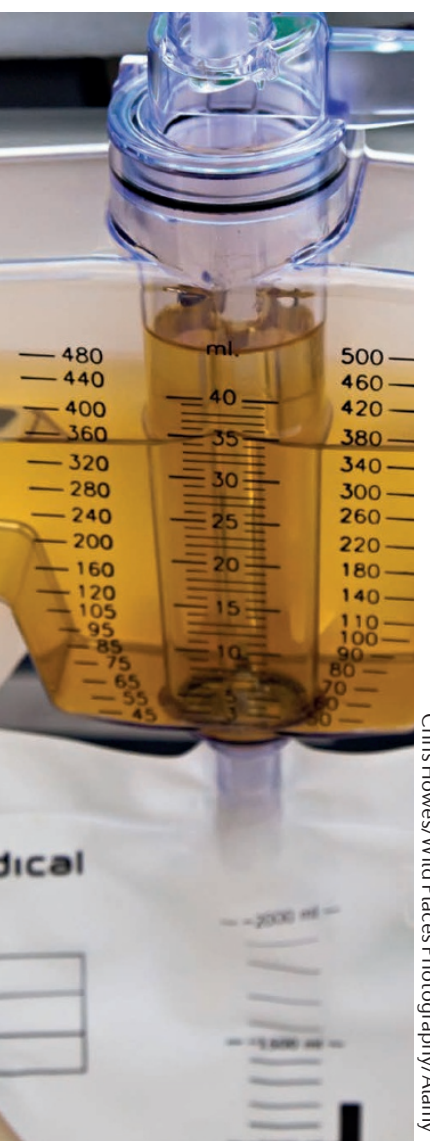

$\Rightarrow$ INFECTION

\section{Proceed with CAUTIon}

Two recent papers in the American Journal of Infection Control have focussed on the changing epidemiology of catheter-associated urinary tract infection (CAUTI).

The first investigated whether the rates of CAUTI have changed since 2011, when public reporting was implemented. Metersky et al. used the Medicare Patient Safety Monitoring System (MPSMS) database to select hospital records from 2009-2014, restricting their sample to patients aged $\geq 18$ years, with acute myocardial infarction (AMI), heart failure, or pneumonia, who had undergone major surgery. The resulting sample included 69,369 patients who had been catheterized whilst an inpatient. During the study period, catheterization frequency decreased significantly $(P<0.001$ for linear trend for AMI, heart failure, or surgical patients). Patients with pneumonia demonstrated a nonsignificant increase in catheterization during the same period. Statistically significant changes in CAUTI rates were observed in patients with AMI and recent surgery only, of $9.7 \%$ and $9.1 \%$ year on year, respectively.

In a second study, Sabir and colleagues sought to determine the incidence of biofilm-based CAUTI and further investigate the aetiology of this condition. Biofilms - microbial communities encased within a self-produced polymeric matrix that adhere to surfaces - are responsible for many difficult-to-treat, persistent, and recurrent infections.

The patient sample included those who had developed $\geq 2$ symptoms of UTI after $\geq 2$ days of indwelling catheterization. Gram staining, colony morphology, and biochemical testing were used to identify the causative pathogens, and tube adherence was used to detect biofilms. $73.4 \%$ of urine isolates were positive for biofilms, detected after a median catheterization period of $5.01 \pm 1.31$ days. Of these, $69.5 \%$ were associated with a latex catheter and $30.4 \%$ with a silicone catheter. The most common causative pathogen was Escherichia coli (52.3\%), but biofilm was most commonly associated with Enterobacter cloacae. Notably, $100 \%$ of biofilm-producing bacteria were ampicillin-resistant, whereas only $17.2 \%$ were resistant to fosfomycin.

Although CAUTI rates seem to be falling, the high abundance of ampicillin-resistant, biofilmproducing pathogens means that tailored intervention strategies are needed.

Annette Fenner

ORIGINAL ARTICLES Metersky, M. L. et al.

National trends in the frequency of bladder catheterization and physician-diagnosed catheter-associated urinary tract infections: results from the Medicare Patient Safety Monitoring System. Am. J. Infect. Control http:// dx.doi.org/10.1016/j.ajic.2017.03.008 (2017)|Sabir, N. et al. Bacterial biofilm-based catheter-associated urinary tract infections: causative pathogens and antibiotic resistance. Am. J. Infect. Control http://dx.doi.org/10.1016/ j.ajic. 2017.05.009 (2017) 